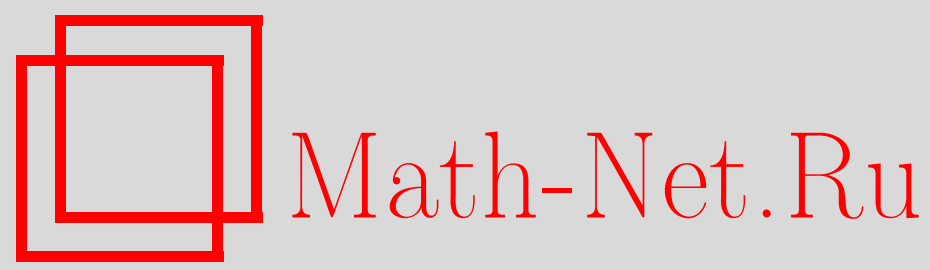

В. П. Маслов, Эконофизика и квантовая статистика, Мaтем. заметки, 2002, том 72, выпуск 6, 883-891

DOI: https://doi.org/10.4213/mzm474

Использование Общероссийского математического портала Math-Net.Ru подразумевает, что вы прочитали и согласны с пользовательским соглашением http://www.mathnet.ru/rus/agreement

Параметры загрузки:

IP : 52.90 .164 .192

26 апреля 2023 г., 12:38:45

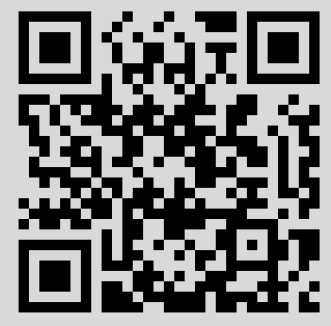




\section{ЭКОНОФИЗИКА И КВАНТОВАЯ СТАТИСТИКА}

\section{В.П. Маслов}

В статье водятся понятия энтропии, температуры, свободной энергии и гамильтониана в задачах теории вероятностей для тождественных объектов так, чтобы можно было применить новейшие методы квантовой статистики к финансовым задачам.

Библиография: 7 названий.

В настоящее время в экономике зародилось новое течение, так назьваемая эконофизика. Дело в том, что ученые стали интуитивно чувствовать аналогии между термодинамикой и экономикой. Для понимания концепций эконофизики, как нам кажется, важно прежде всего обратиться к теории вероятностей и попытаться ввести там некоторые общие понятия, которые в частности приводили бы к термодинамическим законам, и объекты, которые позволяли бы рассматривать в едином ключе квантовую статистику и математическую статистику. Ниже мы введем понятие энтропии и гамильтониана для стандартных задач теории вероятностей.

Рассмотрим случайную величину $Y$

$$
Y_{N,\left\{K_{i}\right\}}=\sum_{i=1}^{n} x_{i} K_{i}, \quad \sum_{i=1}^{n} K_{i}=N
$$

где $K_{i}$ - положительные целые числа, а $x_{i}-$ значения случайной величины $X$, и введем энтропию Больимана как случайную величину, принимающую значения

$$
S_{N,\left\{K_{i}\right\}}=\ln \left\{\frac{N ! \prod_{i=1}^{n} g_{i}^{K_{i}}}{\prod_{i=1}^{n} K_{i} !}\right\}
$$

где $g_{i}$ - целые числа, $\sum_{i=1}^{n} g_{i}=M, g_{i} / M$-вероятности значений $x_{i}$ случайной величины $X$. Пусть $P_{N,\left\{K_{i}\right\}}$-произвольные вероятности, отвечающие случайной величине $Y$, и $\sum P_{N,\left\{K_{i}\right\}}=1$; суммы здесь и далее берутся по всем $\left\{K_{i}\right\}$ таким, что $\sum K_{i}=N$.

Мы определим случайную величину $S$ называемую энтропией: значения $S_{i}$ определяются как экстремумы математического ожидания энтропии Больцмана по всем $P_{N,\left\{K_{i}\right\}}$ при условии, что математическое ожидание случайной величины $Y$ равно константе. С точностью до нормировки вероятности, отвечаюшие этим значениям, равны всевозможным математическим ожиданиям $S_{N,\left\{K_{i}\right\}}$ по соответствуюшим экстремальным значениям $P_{N,\left\{K_{i}\right\}}$. 
Аналогично, полагая $\mathscr{M}(S)=\mathrm{const}$, ищем экстремумы $\mathscr{M}(Y)$. Случайную величину, принимающую эти значения с соответствующими вероятностями, будем назьвать внутренней энергией.

Применяя метод неопределенных множителей Лагранжа, получим экстремум математического ожидания величины

$$
F(\theta)=Y-\theta S
$$

где множитель Лагранжа $\theta$ назьвается температурой.

Точки экстремума математического ожидания величины $F(\theta)(3)$ в общем положении могут быть пронумерованы мультииндексом $l=\left(l_{1}, \ldots, l_{n}\right), l_{\alpha}$ - целые числа, для которых выполняется условие $\sum_{\alpha=1}^{n} l_{\alpha}=N$. Эти экстремумы имеют вид

$$
\sqrt{P}_{N\{K\}}=\prod_{\alpha=1}^{n} \delta_{K_{\alpha} l_{\alpha}}, \quad f^{l}(\theta)=Y_{l}-\theta S_{l}
$$

и называются значениями свободной энергии.

Теорема 1. Выполняется соотношение

$$
\lim _{N \rightarrow \infty}\left(\frac{\min _{l} f^{l}(\theta)}{N}\right)=-\theta \ln \left(\sum_{\alpha=1}^{n} g_{\alpha} e^{-x_{\alpha} / \theta}\right)
$$

где $\min _{l}$ обозначает минимальное из всех значений (4) при фиксированном $\theta$.

Пусть далее $I$ - подмножество набора чисел $\alpha=1,2, \ldots, n$, не равное всему набору. Рассмотрим целое число $C>0$ такое, что существует хотя бы один мультииндекс $K$, удовлетворяюший условиям

$$
\begin{gathered}
K_{\alpha}=0, \text { если } \alpha \in I, \\
\sum_{\alpha \notin I}\left|K_{\alpha}-K_{\alpha}^{I}(\theta)\right|<C,
\end{gathered}
$$

где числа $K_{\alpha}^{I}(\theta)$ имеют вид

$$
\begin{array}{ll}
K_{\alpha}^{I}(\theta)=\frac{e^{-x_{\alpha} / \theta}}{\sum_{\beta \notin I} e^{-x_{\beta} / \theta}} N, & \text { если } \alpha \notin I, \\
K_{\alpha}^{I}(\theta)=0, & \text { если } \alpha \in I .
\end{array}
$$

Будем говорить, что те значения из $f^{l}(\theta)$ вида (4), для которых выполняются условия (6) и (7), принадлежат $\{C, I\}$-окрестности. Справедлива 
Теорема 2. Для значений $f^{l}(\theta)$, принадлежсащих $\{C, I\}$-окрестности, справедливо неравенство

$$
f^{l}(\theta) \geqslant-N \theta \ln \left(\sum_{\alpha \notin I} g_{\alpha} e^{-x_{\alpha} / \theta}\right)
$$

Правую часть неравенства (9) будем назьвать предельной точкой свободной әнергиน.

Рассмотрим задачу о нахождении экстремальных значений математического ожидания $\mathscr{M}(Y)$ случайной величины $Y$ при дополнительном условии

$$
\mathscr{M}(S)=S_{0}
$$

где $S_{0}$ - постоянная, $\mathscr{M}(S)$ - математическое ожидание больцмановской энтропии $S_{N,\left\{K_{i}\right\}}$. Эта задача легко решается методом Лагранжа. Каждой паре мультииндексов $l$ и $m$ таких, что $S_{N,\{m\}} \leqslant S_{0} \leqslant S_{N,\{l\}}$, соответствует экстремальное значение

$$
\tilde{Y}_{\{l\},\{m\}}=Y_{N,\{m\}} \frac{S_{N,\{l\}}-S_{0}}{S_{N,\{l\}}-S_{N,\{m\}}}+Y_{N,\{l\}} \frac{S_{0}-S_{N,\{m\}}}{S_{N,\{l\}}-S_{N,\{m\}}},
$$

соответствующие полуплотности имеют вид

$$
\sqrt{P}_{N,\{K\}}=\delta_{\{K\},\{m\}} \sqrt{\frac{S_{N,\{l\}}-S_{0}}{S_{N,\{l\}}-S_{N,\{m\}}}}+\delta_{\{K\},\{l\}} \sqrt{\frac{S_{0}-S_{N,\{m\}}}{S_{N,\{l\}}-S_{N,\{m\}}}}
$$

и соответствующее значение множителя Лагранжа

$$
\theta_{\{l\},\{m\}}=\frac{Y_{N,\{l\}}-Y_{N,\{m\}}}{S_{N,\{l\}}-S_{N,\{m\}}}
$$

Пусть $\{l\}$ и $\{m\}$ - мультииндексы, для которых вьполнено условие (6) для некоторого $I$. Будем говорить, что соответствующие значения (11) лежат в I-ожрестности. При $N \rightarrow \infty, S_{0} / N \rightarrow S_{0}=$ const сушествуют пределы $\widetilde{Y}_{I}\left(S_{0}\right)=\lim (\min \tilde{Y})$ и $\theta_{I}\left(S_{0}\right)=$ $\lim \widetilde{\theta}$, где $\min \widetilde{Y}$-минимальное из всех значений $(11)$, принадлежащих $I$-окрестности, $\widetilde{\theta}-$ множитель Лагранжа, соответствующий минимальному из принадлежащих $I$-окрестности значению (11).

Справедлива

Теорема 3. 1) Имеет место равенство $\widetilde{Y}_{I}\left(S_{0}\right)-\theta_{I}\left(S_{0}\right) S_{0}=f_{I}\left(\theta_{I}\left(S_{0}\right)\right)$, где $f_{I}(\theta)$ - функция $\theta$ и I такая, что $N f_{I}(\theta)$ совпадает с предельной точкой, определяемой правой частью неравенства (9).

2) Функиия $\theta_{I}(S)$ имеет обратную функиию $S_{I}(\theta)$, причем

$$
\begin{gathered}
S_{I}(\theta)=-\frac{\partial F_{I}(\theta)}{\partial \theta}, \\
\lim _{\theta \rightarrow 0} S_{I}(\theta)=0 .
\end{gathered}
$$


Формула (14) известна в термодинамике, где выражает энтропию через свободную энергию, а (15) совпадает с утверждением теоремы Нернста о стремлении энтропии к нулю при стремлении к нулю температуры.

Рассмотрим теперь аналогичную задачу о нахождении экстремумов математического ожидания $\mathscr{M}(S)$ при дополнительном условии

$$
\mathscr{M}(Y)=Y_{0},
$$

где $Y_{0}$ - постоянная. Экстремальные значения математического ожидания $\mathscr{M}(S)$ имеют вид

$$
\widetilde{S}_{\{l\},\{m\}}=S_{N,\{m\}} \frac{Y_{N,\{l\}}-Y_{0}}{Y_{N,\{l\}}-Y_{N,\{m\}}}+S_{N,\{l\}} \frac{Y_{0}-Y_{N,\{m\}}}{Y_{N,\{l\}}-Y_{N,\{m\}}},
$$

где $\{l\},\{m\}$ - мультииндексы такие, что $Y_{N,\{m\}} \leqslant Y_{0} \leqslant Y_{N,\{l\}}$, соответствующие полуплотности и множитель Лагранжа имеют вид

$$
\begin{aligned}
\sqrt{P}_{N,\{K\}} & =\delta_{\{K\},\{m\}} \sqrt{\frac{Y_{N,\{l\}}-Y_{0}}{Y_{N,\{l\}}-Y_{N,\{m\}}}}+\delta_{\{K\},\{l\}} \sqrt{\frac{Y_{0}-Y_{N,\{m\}}}{Y_{N,\{l\}}-Y_{N,\{m\}}}}, \\
\beta_{\{l\},\{m\}} & =\frac{S_{N,\{l\}}-S_{N,\{m\}}}{Y_{N,\{l\}}-Y_{N,\{m\}}} .
\end{aligned}
$$

Будем говорить, что значение $\widetilde{S}_{\{l\},\{m\}}$ вида (17) лежит в I-полуплоскости, если для мультииндексов $\{l\}$ и $\{m\}$ вьполнено условие (6). При $N \rightarrow \infty, Y_{0} / N \rightarrow \varepsilon_{0}=$ const существуют пределы $\widetilde{S}=\widetilde{S}_{I}\left(\varepsilon_{0}\right)$ и $\theta_{I}\left(\varepsilon_{0}\right)=\lim 1 / \widetilde{\beta}$, где $\max \widetilde{S}$ - максимальное из всех значений (17), принадлежащих $I$-окрестности, а $\widetilde{\beta}$ - соответствующее $\max \widetilde{S}$ значение множителя Лагранжа (19). Имеет место

Теорема 4. 1) Выполнено равенство $\varepsilon_{0}-\theta_{I}\left(\varepsilon_{0}\right) \widetilde{S}_{I}\left(\varepsilon_{0}\right)=f_{I}\left(\theta\left(\varepsilon_{0}\right)\right)$.

2) Выполнено равенство $\widetilde{S}_{I}^{\prime}\left(\varepsilon_{0}\right)=1 / \theta_{I}\left(\varepsilon_{0}\right)$.

3) Функиия $\theta_{I}\left(\varepsilon_{0}\right)$ имеет обратную функиию $\mathscr{E}_{I}(\theta)$, причем справедливо равенcmво $\widetilde{S}_{I}\left(\mathscr{E}_{I}(\theta)\right)=S_{I}(\theta)$.

Таким образом, мы нашли свободную энергию, внутреннюю энергию и энтропию как случайные величины со значениями такими, что предельные точки, индуцируемые предельной точкой свободной энергии, совпадают с термодинамикой в квантовой статистике [1]. Это простейший случай квантования этих переменных, т.е. квантования термодинамики, если случайная величина $X$ ассоциируется с самосопряженным гамильтонианом $\widehat{H}$, а ее значения ассоциируются с собственньми значениями $\widehat{H}$. В нашем случае независимые испытания соответствуют элементам Гиббсовского ансамбля. Предельные точки соответствуют метастабильным состояниям неравновесной термодинамики, в частности, таким как сверхтекучесть и сверхпроводимость [2]. Метод решения таких задач - это метод ультратретичного квантования [3].

Рассматрим ситуацию, когда соответствующая значению случайной величины $x_{j}$ вероятность есть

$$
p_{j}=g_{i} / M
$$


Схема сопоставления вероятностной задаче гильбертова пространства и оператора состоит в следуюшем. Пусть в серии из $N$ испытаний случайная величина $X$ имеет $n$ значений $x_{1}, \ldots, x_{n}$, гильбертово пространство для одного испытания есть $C^{n M}, \mathrm{a}$ оператор $\widehat{H}_{1}$ задается матрищей размера $n M \times n M$, у которой на главной диагонали стоят числа $x_{1}, \ldots, x_{n}$, повторенные $g_{1}, \ldots, g_{n}$ раз (то есть кратности $x_{i}$ равны $g_{i}$ ), а остальные элементы равны 0 . Обозначим через $l_{k}, k=1, \ldots, n$, его собственные векторы. Гильбертово пространство, отвечающее серии из $N$ испытаний, в этом случае есть $C^{n N}$, а гамильтониан $\widehat{H}_{N}$ выражается через $\widehat{H}_{1}$ по формуле

$$
\widehat{H}_{N}=\sum_{s=1}^{N} \widehat{E} \otimes \cdots \otimes \widehat{E} \otimes \widehat{H}_{1} \otimes \widehat{E} \otimes \cdots \otimes E
$$

где $\widehat{E}$ - единичный оператор в пространстве $C^{n}$, каждое слагаемое представляет собой тензорное произведение $N-1$ операторов $\widehat{E}$ и оператора $\widehat{H}_{1}$, причем в слагаемом с номером $s$ оператор $\widehat{H}_{1}$ в произведении стоит на $s$-м месте. Собственные векторы оператора $\widehat{H}_{N}$ имеют вид

$$
f_{k_{1}, \ldots, k_{N}}=e_{k_{1}} \otimes e_{k_{2}} \otimes \cdots \otimes e_{k_{N}}
$$

где $k_{i}=1, \ldots, n$, а соответствующие собственные значения суть

$$
Y_{k_{1}, \ldots, k_{N}}=x_{k_{1}}+x_{k_{2}}+\cdots+x_{k_{N}} .
$$

умноженной на Кратность собственного значения $Y=x_{1} N_{1}+\cdots+x_{n} N_{n}$, которая в случае, ког да числа $x_{1}, \ldots, x_{n}$ несоизмеримы, равна

$$
\frac{N ! \prod_{i=1}^{n} g_{i}^{N_{i}}}{N_{1} ! \cdots N_{n} !}
$$

Рассмотрим теперь обобщения вероятностной игры о вьпгрыше в серии из $N$ независимых испытаний. Рассмотрим выигрыш в серии из $N$ испытаний, причем объединим в одну серию все серии, у которых совпадают вьигрьшш, а различие состоит в порядке выпадения исходов $x_{1}, \ldots, x_{n}$. В некотором смысле можно считать, что испытания тождественны и от их перестановки ничего не меняется.

Сопоставим этой новой задаче пространство $C_{S}^{n N M}$, которое является подпространством пространства $C^{n N M}$ и состоит из его элементов, имеющих вид

$$
f=\sum_{m_{1}=1}^{n} \cdots \sum_{m_{N}=1}^{n} f\left(m_{1}, \ldots, m_{N}\right) \cdot e_{m_{1}} \otimes \cdots \otimes e_{m_{N}}
$$

где $f\left(m_{1}, \ldots, m_{N}\right)$ - числовые коэффициенты, симметричные относительно перестановок $m_{s}$ и $m_{r}, s, r=1, \ldots, n, e_{m}$ - собственные векторы оператора $\widehat{H}_{1}$. Пространство $C_{S}^{n N M}$ будем назьвать симметричным подпространством пространства $C^{n N M}$. Соответствуюший оператор есть ограничение введенного выше оператора $\widehat{H}_{N}$ на подпространство $C_{S}^{n N M}$. Легко убедиться, что кратность собственных значений этого оператора совпадают с формулой (54.4) [1] для Бозе газа. 
Рассмотрим еше одну игру в серии из $N$ испытаний. Так же, как в предыдущей задаче, будем считать, что серии с одинаковьп вьпгрьшем, отличающиеся только перестановкой исходов $x_{m_{1}}, x_{m_{2}}, \ldots, x_{m_{N}}, m_{j}=1, \ldots, n$, объединяются в одну серию, а кроме того наложим запрет на серии, в которых хотя бы один из исходов $x_{1}, \ldots, x_{n}$ встречается больше одного раза. Пространством, отвечаюшим такой постановке вероятностной задачи о выигрыше в серии из $N$ испытаний, является $C_{A}^{n N} M-$ подпространство пространства $C^{n N M}$, состоящее из его элементов, представимьх в виде (24), где коэффициенты $f\left(m_{1}, \ldots, m_{N}\right)$ антисимметричны относительно перестановок $m_{s}$ и $m_{r}$, $s, r=1, \ldots, N$. Будем назьвать $C_{A}^{n N M}$ антисимметричным подпространством пространства $C^{n N M}$. Оператором в данном случае является ограничение оператора $\widehat{H}_{N}$ на подпространство $C_{A}^{n N} M$. Так же, как и в предыдущих случаях, кратность собственных значений оператора совпадает с формулой (54.1) [1] для Ферми газа.

Рассмотренные задачи о серии испытаний принципиально отличаются одна от другой: в этих задачах существует различие между собственными функциями оператоpa $\widehat{H}_{N}$. Собственные функции гамильтониана стандартной задачи о вьпгрыше обладают следуюшим свойством: если представить их в виде столбца, состоящего из $n N$ элементов, то квадрат каждого элемента равен сам себе, поскольку в такой записи векторы представляют собой столбцы из 0 и 1 . Поэтому в обычной задаче о серии независимых испытаний полуплотность совпадает с плотностью вероятности. В случае, когда собственные функции принадлежат симметричному $C_{S}^{n N}{ }^{M}$ или антисимметричному $C_{A}^{n N M}$ подпространству, это свойство не выполняется и полуплотность не равна плотности вероятности. Таким образом, введенное пространство и оператор оказались существенными.

Чтобы учесть температуру и энтропию вместо гамильтониана $\widehat{H}$ надо рассматривать унитарно-нелинейньй оператор свободной энергии (см. [2]-[4]) вида

$$
\lambda \Psi_{\alpha}\left(x_{1}, \ldots, x_{N}\right)=\widehat{H}_{N} \Psi_{\alpha}\left(x_{1}, \ldots, x_{N}\right)+\theta \ln \left(\frac{\left\|\Psi_{\alpha}\right\|^{2}}{\sum_{\beta=1}^{s}\left\|\Psi_{\beta}\right\|^{2}}\right) \Psi_{\alpha}\left(x_{1}, \ldots, x_{N}\right),
$$

c $\alpha=1, \ldots, s$.

Рассмотрим систему из $K$ пачек с деньгами, например, рублями. Введем в рассмотрение $h_{1}$ - "квант денег" - копейку. Каждую пачку будем характеризовать количеством копеек в ней $-n$. Предположим сначала, что пачки не обмениваются между собой деньгами, т.е. число копеек в каждой пачке постоянно. Можно сказать в этом случае, что разные пачки друг с другом не взаимодействуют. Будем предполагать, что

$$
p_{n}=\left(1-e^{-\omega h_{1}}\right) e^{-\omega h_{1} n},
$$

где $\omega=$ const, т.е. имеет место геометрическое распределение. Состояния такой системы (различные случаи распределения денег по пачкам) можно описать векторами следующего пространства $\mathscr{L}_{2}$. Элементами $\mathscr{L}_{2}$ являются векторы вида

$$
\Phi=\sum_{n_{1}=0}^{\infty} \cdots \sum_{n_{K}=0}^{\infty} \Phi\left(n_{1}, \ldots, n_{K}\right) \prod_{s=1}^{K} \frac{\left(b_{s}^{+}\right)^{n_{s}}}{\sqrt{n_{s} !}} \Phi_{0}
$$


где $\Phi_{0}$ - вакуумньй вектор пространства $\mathscr{L}_{2}$, a $b_{s}^{+}$- операторы рождения, сопряженные к которым $b_{s}^{-}$- операторы уничтожения, удовлетворяюшие свойствам

$$
\left[b_{s}^{-}, b_{r}^{+}\right]=\delta_{s r}, \quad\left[b_{s}^{ \pm}, b_{r}^{ \pm}\right]=0, \quad b_{s}^{-} \Phi_{0}=0 .
$$

Случаю, когда в пачке 1 находится $n_{1}$ копеек, в пачке $2-n_{2}$ копеек, и т.д., сопоставим вектор пространства $\mathscr{L}_{2}$, т.е. состояние вида

$$
\prod_{s=1}^{K} \frac{\left(b_{s}^{+}\right)^{n_{s}}}{\sqrt{n_{s} !}} \Phi_{0}
$$

Рассматриваемому случаю, когда пачки не обмениваются между собой деньгами, а распределение имеет вид (26), сопоставляется оператор

$$
\widehat{H}_{K}=h_{1} \omega \sum_{s=1}^{K} b_{s}^{+} b_{s}^{-} \text {. }
$$

Это сопоставление отвечает нашему исходному соотношению между распределением вероятностей по дискретной случайной величине и собственным значением оператора [5]. Используя (27), легко убедиться, что векторы (28) являются собственньгми векторами оператора (29) и соответствующее собственное значение

$$
\lambda_{n_{1}, \ldots, n_{K}}=h_{1} \omega \sum_{s=1}^{K} n_{s}
$$

Отметим теперь, что, сопоставив финансовой задачепространство $\mathscr{L}_{2}$ и оператор $\widehat{H}_{K}$, мы можем перенести принцип неопределенности Гейзенберга в финансовые задачи. Необходимость рассмотрения принципа неопределенности в экономических задачах была отмечена Дж. Соросом [6]. Действительно, рассмотрим операторы

$$
\widehat{Q}=\frac{1}{\sqrt{2}} \sum_{s=1}^{K}\left(b_{s}^{-}+b_{s}^{+}\right), \quad \widehat{P}=\frac{1}{i \sqrt{2}} \sum_{s=1}^{K}\left(b_{s}^{-}-b_{s}^{+}\right) .
$$

Эти операторы самосопряженные, и их коммутатор в силу (27) имеет вид

$$
[\widehat{Q}, \widehat{P}]=i K
$$

Поэтому справедливо следующее неравенство, полностью аналогичное неравенству Гейзенберга: для любого вектора $\Phi \in \mathscr{L}_{2}$

$$
D Q(\Phi) \cdot D P(\Phi) \geqslant \frac{K^{2}}{4}
$$

где

$$
D Q(\Phi)=\frac{\left(\Phi, \widehat{Q}^{2} \Phi\right)}{(\Phi, \Phi)}-\left(\frac{(\Phi, \widehat{Q} \Phi)}{(\Phi, \Phi)}\right)^{2}, \quad D P(\Phi)=\frac{\left(\Phi, \widehat{P}^{2} \Phi\right)}{(\Phi, \Phi)}-\left(\frac{(\Phi, \widehat{P} \Phi)}{(\Phi, \Phi)}\right)^{2}
$$


Заметим, что векторы пространства $\mathscr{L}_{2}$ можно также интерпретировать как состояния системы из $K$ частиц ("больших"), каждая из которых обладает еще внутренней структурой - внутри этих “больших" частищ могут рождаться и уничтожаться "малые" частицы, которым соответствуют операторы $b^{+}$и $b^{-}$. В таком случае оператор (29) - гамильтониан системы невзаимодействующих частищ, а (26) - вероятность того, что "большая" частица состоит из $n$ "малых" частиц. В случае, если частицы взаимодействуют, оператор (29) заменяется на оператор $\widehat{H}_{K}+\widehat{V}_{K}, \widehat{V}_{K}$ - оператор взаимодействия. В физике, как правило, рассматривается парное взаимодействие между частицами. Общий вид оператора парного взаимодействия, сохраняющего симметрию относительно "больших" частиц есть

$$
\widehat{V}_{2 K}=\frac{1}{2} \sum_{s=1}^{K} \sum_{r=1, r \neq s}^{K} f_{2}\left(\stackrel{2}{b}+\stackrel{2}{b_{r}^{+}}, \stackrel{1}{b}-\stackrel{1}{r},-\bar{s}-\right.
$$

где функция $f_{2}\left(z_{1}, z_{2}, w_{1}, w_{2}\right)$ - аналитическая функция по переменным $z_{1}, z_{2}, w_{1}, w_{2}$, а числа над операторами обозначают порядок их действия. Если же взаимодействие $l$-частичное, то соответствующий оператор имеет вид

$$
\widehat{V}_{l K}=\frac{1}{l !} \sum_{s_{1} \neq s_{2} \neq \cdots \neq s_{l}}^{K} f_{l}\left(\stackrel{2}{b}_{s_{1}}^{+}, \ldots, \stackrel{2}{b}_{s_{l}}^{+}, \stackrel{1}{b}_{s_{l}}^{-}, \ldots, \stackrel{1}{b}_{s_{1}}^{-}\right),
$$

где $f_{l}$ - аналитическая функция своих аргументов. Возвращаясь к финансовой задаче, отметим, что оператор (33) можно интерпретировать как переход денег из одной пачки в другую. Действительно, в простейшем частном случае $\widehat{V}_{2 K}$ имеет вид

$$
\frac{1}{2} \sum_{s=1}^{K} \sum_{r=1, r \neq s}^{K} b_{s}^{+} b_{r}^{-}
$$

Этот оператор есть сумма слагаемых $b_{s}^{+} b_{r}^{-}, s \neq r$; под действием такого слагаемого вектор (28) переходит в вектор

$$
\frac{\left(b_{s}^{+}\right)^{n_{s}+1}}{\sqrt{n_{s} !}} \sqrt{n_{r}} \frac{\left(b_{r}^{+}\right)^{n_{r}-1}}{\sqrt{\left(n_{r}-1\right) !}} \prod_{q=1, q \neq s, r}^{K} \frac{\left(b_{q}^{+}\right)^{n_{q}}}{\sqrt{n_{q} !}} \Phi_{0},
$$

т.е. состояние, в котором в пачке $s$ находится $n_{s}$ копеек, а в пачке $r-n_{r}$ копеек, переходит в состояние с $n_{s}+1$ и $n_{r}-1$ копеек в соответствующей пачке. Естественно, операторы $\widehat{V}_{2 K}$ общего вида приводят к тому, что из одной пачки в другую может переходить более, чем одна копейка, а также деньги в пачках могут исчезать и появляться. Операторы (34) в финансовом случае интерпретируются аналогично, только взаимодействие происходит между $l$ пачками с деньгами.

Асимптотика при $N \rightarrow \infty$ приводит, как известно, к уравнению Гросса-Питаевского, или, как его часто называют, к уравнению Хартри - унитарно нелинейному оператору вида $\widehat{H}_{K}+\widehat{V}_{2 K}$, где просто вместо операторов $b^{+}$и $b^{-}$стоят коммутирующие функции $b^{*}$ и $b$. Это унитарно нелинейное уравнение имеет "спектр" и “собственные функции" (см. [2]). Будем назьвать квадрат собственной функции, отвечающей минимальному “собственному значению", одетым распределением. Он отвечает вероятности с 
учетом парного взаимодействия. Квадрат собственной функции оператора $\widehat{H}$ без взаимодействия в $x$-представлении является распределением Гаусса. Одетое распределение может быть найдено экспериментально. Тогда потенциал парного взаимодействия может быть определен из интегрального уравнения первого рода.

Температура в физике по теореме Найквиста пропорциональна дисперсии. Мы предполагаем, что в финансовьх задачах это также имеет место: исходные данные - число пачек денежных купюр - задается неточно и имеет некоторую дисперсию, которую мы будем считать температурой. В этом случаевместо гамильтониана $\widehat{H}_{N}$ нужно рассматривать оператор свободной энергии (25) (см. [2], [4]) и, зная потенциал взаимодействия, вычислить, в частности, зависимость распределения от температуры, а также фазовые переходы, которые естественно считать некоторыми катастрофическими скачками.

Автор выражает благодарность В.Н. Батурину и С. Г. Лебедеву за постановку финансовых задач, которые привели к переосмыслению с позиций квантовой статистики задач теории вероятностей.

\section{СПИСОК ЦИТИРОВАННОЙ ЛИТЕРАТУРЫ}

[1] Ландау Л. Д., Лифшиц Е. М. Статистическая физика. М.: Наука, 1976.

[2] Маслов В. П. Квантование термодинамики и ультравторичное квантование. М.: Институт компьютерных исследований, 2001.

[3] Маслов В. П. Ультратретичное квантование термодинамики // ТМФ. 2002. Т. 132. №3. C. $388-398$.

[4] Маслов В. П. Квантование больцмановской энтропии, пары и корреляционная функция // ТМФ. 2002. Т. 131. №2. С. 261-277.

[5] Маслов В. П. Элементарная проквантованная термодинамика в стандартных вероятностных схемах // Докл. РАН. 2002. Т. 385. № 4. С. 456-459.

[6] Soros G. The dangers of capitalism // The Atlantic Monthly. 1997. V. 279. № 2. P. 45-58.

Московский государственный университет им. М.В. Ломоносова

Поступило

E-mail: maslov@qs.phys.msu.su

05.08.2002

Исправленный вариант

25.10.2002 